\title{
Perfil epidemiológico dos casos de tuberculose no estado do Maranhão nos anos de 2012 a 2016
} Epidemiological profile of tuberculosis cases in the state of Maranhão in the years of 2012 to 2016

Perfil epidemiológico de los casos de tuberculosis en el estado de maranhão en los años de 2012 a 2016

Mara Sílvia Rocha Oliveira ${ }^{1}$, Luiza Cristiny Sousa ${ }^{1}$, Luciana Stanford Baldoino ${ }^{2}$, Alda Alves Alvarenga ${ }^{1}$, Maria Nauside Pessoa da Silva ${ }^{1,3}$, Stefania da Costa Gomes Elias ${ }^{1}$, Luciana Soares Macedo ${ }^{1}$, Mara Ramel de Sousa Silva ${ }^{1,4}$

1. Departamento de Enfermagem, Instituto de Ensino Superior Múltiplo, Timon, Maranhão, Brasil.

2. Mestrado em Ciências da Educação, Universidade de Campinas, Campinas, São Paulo, Brasil.

3. Programa de Pós-Graduação em Biotecnologia (RENORBIO), Universidade Estadual do Ceará, Fortaleza, Ceará, Brasil

4. Programa de Pós-graduação em Ciência Animal, Universidade Federal do Piauí, Teresina, Piauí, Brasil.

\section{ABSTRACT}

Objective: to analyze the epidemiological profile of tuberculosis in the state of Maranhão, Brazil, from 2012 to 2016. Methodology: This is an epidemiological, descriptive, retrospective study, using cases reported by DATASUS concerning tuberculosis diseases diagnosed in residents in the state of Maranhão between 2012 and 2016. The variables studied were: race, sex, age, schooling, municipality of residence, year of diagnosis and treatment. Results: Tuberculosis cases were predominantly found in individuals aged between 20 and 39 years (43.1\%), with incomplete elementary school (68.5\%), brown (70.5\%) and male sex (64.7\%). Conclusion: The reported incidence rates of tuberculosis in the State of Maranhão during the years 2012 to 2016 had small oscillations. It has been observed that tuberculosis is a persistent public health problem.

Descriptors: Notified Tuberculosis; Public health; Epidemiological Profile.

\section{RESUMO}

Objetivo: analisar o perfil epidemiológico da tuberculose no estado do Maranhão, Brasil de 2012 a 2016. Metodologia: Trata-se de um estudo epidemiológico, descritivo, de coleta retrospectiva, por meio de casos notificados pelo DATASUS referentes aos agravos de tuberculose diagnosticados em residentes no estado do Maranhão entre 2012 a 2016. As variáveis estudadas foram: raça, sexo, idade, escolaridade, município de residência, ano de diagnóstico e o tratamento. Resultados: Observou-se que os casos de tuberculose estiveram predominantemente em indivíduos com faixa etária de 20 a 39 anos $(43,1 \%)$, com ensino fundamental incompleto $(68,5 \%)$, de raça parda $(70,5 \%)$ e sexo masculino $(64,7 \%)$. Conclusão: As taxas de incidência notificadas de tuberculose no Estado do Maranhão durante os anos 2012 a 2016 tiveram pequenas oscilações. Observou-se que a tuberculose é um persistente problema de saúde pública.

Descritores: Tuberculose Notificada; Saúde Pública; Perfil Epidemiológico.

\section{RESUMÉN}

Objetivo: analizar el perfil epidemiológico de la tuberculosis en el estado de Maranhão, Brasil de 2012 a 2016. Metodología: Se trata de un estudio epidemiológico, descriptivo, de recolección retrospectiva, por medio de casos notificados por el DATASUS referentes a los agravios de tuberculosis diagnosticados en residentes en el estado de Maranhão entre 2012 y 2016. Las variables estudiadas fueron: raza, sexo, edad, escolaridad, municipio de residencia, año de diagnóstico y el tratamiento. Resultados: Se observó que los casos de tuberculosis estuvieron predominantemente en individuos con edades de 20 a 39 años $(43,1 \%)$, con enseñanza básica incompleta $(68,5 \%)$, de raza parda $(70,5 \%)$, sexo masculino $(64,7 \%)$. Conclusión: Las tasas de incidencia notificadas de tuberculosis en el Estado de Maranhão durante los años 2012 a 2016 tuvieron pequeñas oscilaciones. Se observó que la tuberculosis es un problema persistente de salud pública.

Descriptores: Tuberculosis notificada; Salud pública; Perfil Epidemiológico.

\section{Como citar este artigo:}

Oliveira MSR, Sousa LC, Baldoino LS, Alvarenga AA, Silva ANP, Elias SCG, et al. Perfil Epidemiológico dos Casos de Tuberculose no Estado do Maranhão nos Anos de 2012 a 2016. Rev Pre Infec e Saúde[Internet]. 2018;4:6896.

Available from: http://www.ojs.ufpi.br/index.php/nupcis/article/view/6896

DOI: https://doi.org/10.26694/repis.v4i0.6896

Rev Pre Infec e Saúde.2018;4:6896 


\section{INTRODUÇÃO}

A tuberculose (TB) é uma doença infectocontagiosa causada pelo Mycobacterium tuberculosis ou Bacilo de Koch (BK), acometendo muita das vezes os pulmões e podendo levar a morte. A TB tem tratamento, e por meio dele, pode-se atingir a cura do paciente, medidas simples e certas podem evitar a transmissão de pessoa a pessoa ${ }^{1}$.

Em 2014, durante a Assembleia Mundial de Saúde, na Organização Mundial de Saúde, foi aprovada a nova estratégia global para enfrentamento da tuberculose, com a visão de um mundo livre da tuberculose até 2035. O Brasil teve o papel de destaque ao ser o principal proponente da estratégia e principalmente por sua experiência com o Sistema Único de Saúde e com a Rede de Pesquisa em Tuberculose do Brasil (Rede-TB) ${ }^{2}$.

Nos últimos dez anos, o Brasil reduziu em 22,8\% a incidência de casos novos de TB e em 20,7\% à taxa de mortalidade da doença. Em 2014, a incidência da doença no Brasil foi de 33,5 casos por 100 mil habitantes, contra 43,4/100 mil em 20043.

O Brasil faz parte do grupo dos 22 países de alta carga priorizados pela Organização Mundial da Saúde (OMS), que concentram $80 \%$ dos casos de tuberculose no mundo, ocupando a $16^{\mathrm{a}}$ posição em número absoluto de casos (BRASIL, 2015). Em 2013 dentre os Estados do Nordeste o Maranhão ocupava em sexto lugar as maiores taxas de incidências de TB por 100 mil habitantes ${ }^{4}$.

De acordo com a Vigilância Sanitária Estadual Açailândia, Caxias, Timon, Imperatriz, São Luís, São José de Ribamar, Paço do Lumiar e Codó são municípios que têm mais de 100 mil habitantes, e que têm uma carga de incidência da doença que ainda preocupa. No Maranhão, a média é de 2.400 casos de tuberculose registrados por ano. A capital está entre as oito cidades com maior incidência de casos de TB no Estado3.

Dessa forma, este estudo tem como objetivo caracterizar o perfil da tuberculose no estado do Maranhão entre os anos de 2012 a 2016.

\section{MÉTODOS}

Trata-se de um estudo epidemiológico, descritivo, de coleta retrospectiva, por meio de casos notificados pelo DATASUS referentes aos agravos de tuberculose diagnosticados em residentes no Estado do Maranhão entre 2012 a 2016.

A coleta de dados foi realizada por meio de tabuladores disponibilizados pelo Ministério da Saúde: TABNET, seguindo os seguintes passos: 1) acesso ao link http://dtr2004.saude.gov.br/sinanweb/; clicar em tabulador de dados; c) selecionar agravo que deseja pesquisar- tuberculose; d) acessos aos dados de acordo com as variáveis citadas acima.

Realizou-se cruzamento de informações em várias variáveis, considerando aspectos quantitativos, como por exemplo, número de paciente, estado da doença, evolução dos casos, dentre outro, possibilitando a resposta ao objetivo proposto.

As análises ocorrem por meio de técnicas descritivas simples, utilização do Excel software Microsoft Office 2010 ferramenta de 
tratamento de dados e análise de estatísticas simples e construção de gráficos. 0 estudo obedeceu a todos os preceitos éticos

\section{RESULTADOS}

Observou-se que no período de 2012 a 2016, no Estado do Maranhão os casos de tuberculose que regem a pesquisa com seres humanos no Brasil.

estavam predominante na faixa etária de 20 a 39 anos $(43,1 \%)$ (tabela 1$)$.

Tabela 1: Distribuição dos casos de tuberculose, segundo as características sócias demográficas no período de 2012-2016. Estado do Maranhão, 2017.

\begin{tabular}{|c|c|c|c|c|c|c|c|c|c|c|c|c|}
\hline \multirow{3}{*}{ Variáveis } & \multicolumn{8}{|c|}{ Ano de Notificação } & & & & \\
\hline & \multicolumn{2}{|c|}{2012} & \multicolumn{2}{|c|}{2013} & \multicolumn{2}{|c|}{2014} & \multicolumn{2}{|c|}{2015} & \multicolumn{2}{|c|}{2016} & \multicolumn{2}{|c|}{ Total } \\
\hline & $n$ & $\%$ & $n$ & $\%$ & $\mathrm{n}$ & $\%$ & $\mathrm{n}$ & $\%$ & $\mathrm{n}$ & $\%$ & $\mathrm{n}$ & $\%$ \\
\hline \multicolumn{13}{|l|}{ Sexo } \\
\hline Masculino & 1431 & 66,2 & 1483 & 63,3 & 1375 & 63,8 & 1479 & 65,5 & 381 & 66,8 & 6149 & 64,7 \\
\hline Feminino & 733 & 33,8 & 861 & 36,7 & 779 & 36,2 & 781 & 34,5 & 189 & 33,2 & 3343 & 35,3 \\
\hline Total & 2164 & 100 & 2344 & 100 & 2154 & 100 & 2260 & 100 & 570 & 100 & 9492 & 100 \\
\hline \multicolumn{13}{|l|}{ Escolaridade } \\
\hline Ignorado/Branco & 97 & 4 & 73 & 3,1 & 88 & 4,1 & 67 & 3 & 4 & 0,7 & 329 & 3,5 \\
\hline $\begin{array}{l}\text { Ensino fundamental } \\
\text { incompleto }\end{array}$ & 1493 & 69 & 1624 & 69 & 1473 & 68,4 & 1525 & 67,5 & 388 & 68 & 6503 & 68,5 \\
\hline Ensino médio completo & 324 & 15 & 357 & 15 & 311 & 14,4 & 357 & 15,8 & 104 & 18,3 & 1453 & 15,3 \\
\hline Ensino médio incompleto & 142 & 7 & 168 & 7,7 & 150 & 6,9 & 176 & 7,8 & 45 & 8 & 681 & 7,2 \\
\hline Educação superior completa & 78 & 3,6 & 85 & 3,6 & 96 & 4,5 & 95 & 4,2 & 23 & 4 & 377 & 4 \\
\hline Não se aplica & 30 & 1,4 & 37 & 1,6 & 36 & 1,7 & 40 & 1,7 & 6 & 1 & 149 & 1,5 \\
\hline Total & 2164 & 100 & 2344 & 100 & 2154 & 100 & 2260 & 100 & 570 & 100 & 9492 & 100 \\
\hline \multicolumn{13}{|l|}{ Raça } \\
\hline Ignorado/Branco & 39 & 1,8 & 33 & 1,4 & 25 & 1,2 & 34 & 1,5 & 11 & 1,9 & 142 & 1,5 \\
\hline Branca & 291 & 13,5 & 279 & 11,9 & 252 & 11,7 & 260 & 11 & 60 & 10,5 & 1142 & 12 \\
\hline Preta & 299 & 13,8 & 313 & 13,4 & 263 & 12,2 & 279 & 13,3 & 81 & 14,2 & 1235 & 13 \\
\hline Amarela & 13 & 0,6 & 16 & 0,7 & 14 & 0,6 & 16 & 0,7 & 2 & 0,3 & 61 & 0,7 \\
\hline Parda & 1485 & 68,6 & 1643 & 70 & 1550 & 72 & 1614 & 71 & 406 & 71,3 & 6698 & 70,5 \\
\hline Indígena & 37 & 1,7 & 60 & 2,6 & 50 & 2,3 & 57 & 2,5 & 10 & 1,8 & 214 & 2,3 \\
\hline \multicolumn{13}{|l|}{ Zona de residência } \\
\hline Ignorado/Branco & 39 & 1,8 & 50 & 2,1 & 58 & 2,5 & 63 & 2,7 & 10 & 1,8 & 220 & 2,2 \\
\hline Urbana & 1483 & 68,5 & 1617 & 69 & 1533 & 71,3 & 1573 & 69,5 & 397 & 69,6 & 6603 & 69,5 \\
\hline Rural & 596 & 27,5 & 632 & 26,9 & 534 & 24,8 & 569 & 25,4 & 161 & 28,3 & 2492 & 26,5 \\
\hline Periurbana & 46 & 2,2 & 45 & 2 & 29 & 1,4 & 55 & 2,4 & 2 & 0,3 & 177 & 1,8 \\
\hline Total & 2164 & 100 & 2344 & 100 & 2154 & 100 & 2260 & 100 & 570 & 100 & 9492 & 100 \\
\hline
\end{tabular}


Quanto ao tipo de entrada, observou-se que $84 \%$ dos casos novos foram de TB, seguido de reingresso após abandono com 7,1\%. 0 número de casos notificados que não sabe e pós-óbito corresponde a 0,2\% cada. E o não informado com $54,2 \%$ apresentou maior número de casos de TB, dar se pela falta de notificação por completo, pessoas que deram entrada, foram admitidos com TB mais, não informaram como entraram no sistema.

Verificou-se ainda que na maioria dos casos de TB a doença teve como sua situação encerrada a cura com 59,4\%. O número de casos transferido foi de $8,1 \%$, já a porcentagem de óbito pela doença foi de $3,6 \%$ e óbito por outras causas foram $2,9 \%$, sendo que em $10 \%$ dos casos ocorreram abandono.

Os casos de tuberculose mantiveram-se com números semelhantes no decorrer do período estudado. Vale ressaltar que no ano de 2016, houve uma queda com relação aos anos anteriores. Os casos notificados nesse ano não estão completos, devido o não fechamento do banco de dados do DATASUS (SINAN/NET) onde foi coletado até o mês de março de 2017 (tabela 2).

Tabela 2: Indicador de Taxa de Incidência de TB no Maranhão, segundo o ano de notificação no período de 2012-2016, Estado do Maranhão, 2017.

\begin{tabular}{|c|c|c|c|c|c|c|c|c|c|c|c|c|c|c|c|c|c|}
\hline & & & & \multicolumn{14}{|c|}{ Ano de Notificação } \\
\hline \multicolumn{2}{|c|}{2012} & \multicolumn{4}{|c|}{2013} & \multicolumn{2}{|c|}{2014} & \multicolumn{3}{|c|}{2015} & \multicolumn{3}{|c|}{2016} & \multicolumn{4}{|c|}{ Total } \\
\hline $\mathrm{n}$ & $\%$ & $\mathbf{i}$ & $\mathbf{n}$ & $\%$ & $\mathbf{i}$ & $\mathbf{n}$ & $\%$ & I & $\mathrm{n}$ & $\%$ & $\mathbf{i}$ & $\mathrm{n}$ & $\%$ & $\mathbf{i}$ & $\mathrm{n}$ & $\%$ & I \\
\hline
\end{tabular}

No período estudado os exames de escarro $87 \%$ e o TRM $28,4 \%$ tiveram o número elevado de não realização do exame, mesmo sendo este um dos métodos de diagnóstico mais efetivo para o tratamento da TB.

\section{DISCUSSÃO}

Neste estudo, foram confirmados e notificados 9.492 casos de Tuberculose no Estado do Maranhão entre os anos de 2012 a 2016 pelo SINAN/NET. Com relação ao perfil sócio demográfico da tuberculose, a faixa etária entre 20 e 39 anos apresentou a maior concentração de casos em relação às outras idades, com

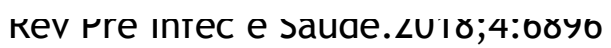

prevalência do sexo masculino, resultados corroborados por outros estudos nacionais ${ }^{5-7}$.

Consumo de álcool, formas de trabalho e a maior procura por parte das mulheres aos serviços de saúde são apontados na literatura como fatores associados à diferença entre os sexos, colaborando para maior prevalência no sexo masculino ${ }^{5-8}$. Quanto à idade, indivíduos da fase economicamente produtiva foram os mais atingidos. A existência de jovens adultos bacilíferos indica a ocorrência de transmissão recente. Esse padrão epidemiológico difere do encontrado em países europeus, em que a doença tem sido mais bem controlada e atinge uma camada mais idosa da população'. Outro 
estudo realizado em Belém, Pará, Brasil, que relata resultados semelhantes no que concerne ao perfil de gênero levanta como hipótese o fato do homem não cuidar adequadamente de sua saúde e ainda estar mais exposto aos fatores de risco para a doença quando comparados às mulheres $^{10}$.

Quanto ao grau de escolaridade, predominou-se 0 ensino fundamental incompleto, como corroborado por outros estudos de delineamento semelhante realizados no Brasil ${ }^{5,11-13}$. O baixo grau de instrução dos pacientes pode influenciar negativamente na aquisição de informações, configurando-se como um fator determinante para o aumento da vulnerabilidade social ao qual o indivíduo está exposto. 0 grau de instrução favorece um maior entendimento e apreensão dos conhecimentos levantados sobre o viver com a doença ou o estar doente. Vale ressaltar que a baixa escolaridade poderia aumentar a vulnerabilidade à TB ao refletir acesso individual e desigual à informação, aos bens de consumo e ao próprio serviço de saúde, funcionando como um marcador de precárias condições de vida e maior vulnerabilidade à $\mathrm{TB}^{11-14}$.

Em nosso estudo o encerramento dos casos por cura foi prevalente, mas também destacou-se alta porcentagem de abandono e óbito por outras causas. Em um outro estudo ${ }^{15}$ realizado no Brasil observou-se que ao final do acompanhamento, a situação de encerramento foi de cura em $68,8 \%$ dos casos, enquanto abandono do tratamento ocorreu em 9,7\% dos casos, óbito do paciente por tuberculose $(2,0 \%)$ ou por outras causas $(6,8 \%)$.
Entretanto, no período estudado os exames de escarro $87 \%$ e o TRM $28,4 \%$ tiveram o número elevado na não realização do exame, mesmo sendo este um dos métodos de diagnóstico mais efetivo para o tratamento da TB.

Registramos elevada porcentagem de não realização dos exames de escarro e Teste Rápido Molecular. Pesquisa realizada no Brasil $^{16}$ corrobora nossos achados, relevando que a cultura não foi realizada na maior parte dos casos $(86,2 \%)$, pelo fato da cultura de escarro, ser considerada um exame complexo e de alto custo comparada com a baciloscopia. Coerentes com o exposto, estudos reafirmam que as vantagens da baciloscopia são o baixo custo, e as desvantagens da cultura é o longo tempo para o resultado.

Tanto a baciloscopia como a cultura para micobactéria com identificação de espécie (CM) e, mais recentemente, o teste rápido molecular para tuberculose (TRM-TB) depende da quantidade e da qualidade da amostra de escarro para que seus resultados sejam confiáveis ${ }^{17}$.

A cultura é o método diagnóstico considerado padrão ouro para confirmação da tuberculose, com elevada sensibilidade e especificidade para 0 diagnóstico da tuberculose. Contudo, é pouco utilizada no processo de tomada de decisão clínica, devido à demora em obtenção do resultado, em virtude da reprodução lenta do bacilo. O TRM-TB apresenta sensibilidade de $88 \%$ e especificidade de $98 \%$. Dessa forma, a utilização do teste, antes da cultura, representa agilidade na confirmação do resultado e o tratamento podendo ser 
Oliveira MSR, et al

iniciado mais rápido, sem a necessidade de se aguardar a confirmação pela cultura ${ }^{18}$.

\section{CONCLUSÃO}

Diante do exposto observou-se que a tuberculose é um persistente problema de saúde pública. Os resultados deste estudo possibilitaram analisar as características dos casos notificados da tuberculose de 2012 a 2016 no Estado do Maranhão, com a confirmação de 9.592 mil casos novos da doença.

Notou-se que as taxas de incidência de notificadas de TB no Estado do Maranhão durante os anos 2012 a 2016 tiveram pequenas oscilações de um período e outro. Apresentando a maior incidência com $(0,23)$ no ano de 2013 e menor incidência $(6,0)$ em 2016. Devido o não encerramento do sistema no banco de dados DATASUS (SINAN/NET) no qual foi coletado até o mês de maio de 2017.

\section{REFERÊNCIAS}

1. Ministério da Saúde. Secretaria de Vigilância em Saúde. Manual de recomendações para o controle da tuberculose no Brasil. 1th ed. Brasília: 2011. Available from: http://bvsms.saude.gov.br/bvs/publicacoes/ma nual_recomendacoes_controle_tuberculose_brasi l.pdf.

2. Ministério da Saúde. Secretaria de Atenção à Saúde. Departamento de Atenção Básica. Plano Nacional pelo Fim da Tuberculose como Problema de Saúde Público. 1th ed. Brasília: 2017.

Available

from:

http://portalarquivos.saude.gov.br/images/pdf /
Perfil epidemiológico dos casos de Tuberculose 2017/fevereiro/24/Plano-Nacional-

Tuberculose.pdf.

3. Ministério da Saúde. Taxa de mortalidade por tuberculose caem 20,7\% em 10 anos. 1th ed. Brasília: 2015. Available from: http: //www.brasil.gov.br/saude/2015/03/taxade-mortalidade-por-tuberculose-cai-20-7-em-10anos.

4. Silva IP, Pontes AN, Silva BC, Silva IP, Bichara CNC. Aspectos Clínicos e Epidemiológicos da Tuberculose no Município de Imperatriz, Maranhão. Enciclopédia Biosfera. 2016; 13(24):1385-99. Available from: http: //www.conhecer.org.br/enciclop/2016b/sa ude/aspectos\%20clinicos.pdf.

5. Reis DN, Almeida TCA, Quites HFO, Sampaio $M M$. Epidemiological profile of tuberculosis in the city of Belo Horizonte (MG), from 2002 to 2008. Rev Bras Epidemiol. 2013; 16(3):592-602. Available from: http://www.scielo.br/scielo.php?script=sci_artt ext\&pid=S1415-

$790 \times 2013000300592 \& \operatorname{lng}=e n \& n r m=i s o \& t \operatorname{lng}=e n \&$ ORIGINALLANG=en.

6. Coutinho LASA, Oliveira DS, Souza GF, Filho GMCF, Saraiva MG. Perfil Epidemiológico da Tuberculose no Município de João Pessoa - PB, entre 2007 - 2010. Rev Bras Cienc Saúde. 2012; 16(1):35-42. Available from: http://periodicos.ufpb.br/index.php/rbcs/articl e/view/10172.

7. Prado TN, Galavote HS, Brioshi AP, Lacerda T, Fregona G, Detoni VV, et al. Epidemiological profile of tuberculosis cases reported among health care workers at the University Hospital in Vitoria, Brazil. J Bras Pneumol. 2008; 34(8):60713. Available from: 
Oliveira MSR, et al

http: / /www.scielo.br/scielo.php?script=sci_artt ext\&pid=S1806-

$37132008000800011 \& \operatorname{lng}=e n \& n r m=i s o \& t \operatorname{lng}=e n$.

8. Monteiro PC, Gazzeta CE. Aspectos epidemiológicos, clínicos e operacionais do controle da tuberculose em um Hospital Escola 1999 a 2004. Arq Ciênc Saúde. 2007; 14(2):99106. Available from: http://repositorioracs.famerp.br/racs_ol/vol-14-2/IIDD180.pdf.

9. Cavalcante EFO, Silva DMGV. Perfil de pessoas acometidas por tuberculose. Rev Rene. 2013; 14(4):720-9. Available from: http://www.periodicos.ufc.br/rene/article/vie $\mathrm{w} / 3531$.

10. Freitas WMTM, Santos CC, Silva MM, Rocha GA. Clinical and epidemiological profile of patients with tuberculosis cared at a municipal health center in Belém, Pará State, Brazil. Rev Pan-Amaz Saude. 2016; 7(2):45-50. Available from:

http://scielo.iec.gov.br/scielo.php?script=sci_ar ttext\&pid=S2176-62232016000200045.

11. Silveira MPT, Adorno RFR, Fontana T. Perfil dos pacientes com tuberculose e avaliação do programa nacional de controle da tuberculose em Bagé (RS). J bras pneumol. [Internet]. 2007; 33(2): 199-205. Available from: http://www.scielo.br/scielo.php?script=sci_artt ext\&pid=S1806-37132007000200015\&lng=en. 12. Araújo KMFA, Figueiredo TMRM, Gomes LCF, Pinto ML, Silva TC, Bertolozzi MR. Evolution of the spatial distribution of tuberculosis cases in the city of Patos (PB), 2001-2010. Cad Saúde Colet. 2013; 21(3):296-302. Available from:

13. Caliari JS, Figueiredo RM. Tuberculosis: patient profile, service flowchart, and nurses' opinions. Acta Paul Enferm. 2012; 25(1):43-7.
Perfil epidemiológico dos casos de Tuberculose Available from: http://www.scielo.br/scielo.php?script=sci_artt ext\&pid=S0103-

$21002012000100008 \& \operatorname{lng}=e n \& n r m=i s o \& t \operatorname{lng}=e n$.

14. Ferreira RCZ, Sobrinho ECR, Zóia EN, Figueiredo RM. Perfil epidemiológico da tuberculose em município do interior paulista (2001- 2010). Cuid Enferm. 2013; 7(1):7-12. Available from: http://pesquisa.bvsalud.org/enfermagem/resour ce/pt/bde-25101.

15. Angellotti LCZ, Alexandre PBD, Miranzi SSC, Scatena LM. Qualidade de Dados de Notificação e Acompanhamento dos Casos de Tuberculose em Minas Gerais. Rev enferm atenção saúde. 2013; 2(2):84-98. Available from: http://seer.uftm.edu.br/revistaeletronica/indeh $\mathrm{p} /$ enfer/article/view/387.

16. Ferri AO, Aguiar B, Wilhelm CM, Schmidt D, Fussieger F, Picoli SU. Diagnóstico de Tuberculose: uma revisão. Revista Liberato. 2014; 15(24):105-212. Available from: http://revista.liberato.com.br/ojs2/index.php/r evista/article/view/317.

17. Ministério da Saúde. Secretaria de Atenção à Saúde. Departamento de Atenção Básica. Programa Nacional de Controle da Tuberculose. 1th ed. Brasilia: 2014. Available from: http://bvsms.saude.gov.br/bvs/publicacoes/Pro gramaTB.pdf.

18. Miranda LO, Araujo GBF, Carvalho ML, Silva SMF, Andrade DFR, Moreira AM, et al. Aspectos epidemiológicos da coinfecção Tuberculose/HIV no Brasil: revisão integrativa. Rev Pre Infec e Saúde. 2017; 3(3):59-70. Available from: http://www.ojs.ufpi.br/index.php/nupcis/articl e/view/5848. 


\section{COLABORAÇÕES}

Oliveira MSR, Sousa LC, Alvarenga AA, Silva ANP, Elias SCG e Baldoino LS, participaram da concepção inicial do projeto de pesquisa, desde a escolha e delineamento do desenho do estudo até a coleta dos dados e interpretação dos resultados iniciais obtidos. Macedo LS e Silva MRS contribuíram com a leitura final e estruturação crítica da redação científica do artigo.

\section{CONFLITOS DE INTERESSE}

Não há conflitos de interesse a declarar

\section{CORRESPONDENCIA}

Luciana Stanford Baldoino

Rua Visconde da Parnaíba, 3377, ap. 404

CEP 64049570 Teresina, PI, Brasil. Telefone: (86) 99978-6956

E-mail: Isbaldoino@hotmail.com 